\title{
Ischemic heart disease and Diabetes mellitus: a statistical analysis
}

Several authors have described diabetes mellitus (DM) as an independent risk factor for ischemic heart disease (IHD). The objective of this study was to test the hypothesis of the association of DM and IHD. Adjustment of the possible variables of confusing and/or modifying effects was performed in the present study. The study also assessed the existence of a doseresponse gradient for the following variables:

- duration of DM;

- duration of elevated blood pressure;

- duration of hypercholesterolemia;

— duration of smoking habits;

— number of cigarettes per day;

- duration of menopause.

The study was a case-control type. The target population was made of subjects aged 30 to 69 years of both genders and residents of São Paulo. The study began March 1993 and it ended in February 1994. Patient sample was 833 subjects. Statistical analysis employed was multivariate logistic regression.

Raw data analysis indicated DM as a risk factor in a statistically significant way for the emergence of IHD (odds ratio $=3.7$; confidence interval $=1.8-7.6$ ) . With the inclusion of variables such as family history of heart disease, smoking habits, hypercholesterolemia and family history of hypertension in the testing model, a non-significant relationship emerges (odds ratio $=1.4$; confidence interval $=0.6-2.3$ ). This change might be explained bythese four variables known to be associated with $I H D$. In the next step, the study tried to segregate the cluster of individuals not belonging to any of the groups above. This was done to determine the degree of strength in the association of DM with a minimal degree of interference by other variables. The greatest shortcoming is the small number of subjects with $\operatorname{DM}(92)$, which is only $11 \%$ of the entire sample. 